\section{GELECHIID DIVERSITY (LEPIDOPTERA) FROM SIWALIK HILLS OF NORTH- WESTERN HIMALAYA}

\author{
P.C. Pathania ${ }^{1}$, H.S. Rose ${ }^{2}$ and Rachita Sood ${ }^{2}$ \\ ${ }^{1}$ Division of Entomology, Indian Agricultural Research Institute, New \\ Delhi 110012, India \\ 2 Department of Zoology, Punjabi University, Patiala, Punjab \\ 1470002, India \\ Email: ${ }^{1}$ pathaniapc@yahoo.co.in
}

According to Chitkara (1998), the elevation of the Siwaliks range extends up to $1500 \mathrm{~m}$ and demarcated between river Kali in Uttaranchal and river Indus in Jammu and Kashmir. During the course of present surveys conducted between 1997 and 2001, we visited localities such as Jammu, Katra, Mansar Lake (Jammu \& Kashmir), Kangra, Palampur, Dharamshala, Tanyhar, Sarkaghat, Baijnath, Andhretta, Solan, Dharampur, Nauni, Sabthu, Renuka lake, Nahan, Paunta Sahib (Himachal Pradesh), Dehradun, Haridwar, Vikasnagar (Uttaranchal), Kalka, Pinjore (Haryana), Pathankot, Hoshiarpur, Dasua, Dhar, Roopnagar, Dunera, Mirzapur, Nawanshahar and Anandpur (Punjab) in the Siwaliks of north-western Himalaya.

Gelechiid moths were collected at night with the help of portable light traps. In addition to this, some specimens were also collected by hanging the source of light (125-Watt mercury vapour lamp) on a white sheet or white washed wall. The newly collected insects were killed using 1,1,2,2, tetrachloro ethane or ethyl acetate. The specimens collected from various localities were processed as per methodology discussed by workers such as Lindquist (1956), Tagestad (1974), Zimmerman (1978), Mikkola (1986) and Landry and Landry (1994). For wing venation standard techniques given by Zimmerman (1978) and for genitalia Robinson (1976) was followed. Various morphological characters (Robinson, 1976; Hodges, 1998; Park, 1995), wing venation (Zimmerman, 1978; Common, 1970) and external genitalia (Klots, 1970) were described. All illustrations were made using a Camera Lucida attached to a stereoscopic light microscope (Nikon SMZ-10, Japan). All specimens were photographed prior to dissection.

The collected material were assigned to various taxa following (Meyrik, (1905, 1907, 1908a, 1908b, 1909, 1910, 1911, 1912-1916, 1913, 1914, 1916-1923, 1923-1930, 1930-1936), Gaede (1937); Clarke (1969a, 1969b); Park and Omelko (1994), Robinson, et al. (1994), Park and Hodges (1995), and Ueda (1995). All specimens deposited in the Lepidoptera Laboratory, Department of Zoology, Punjabi University, Patiala.

\section{Superfamily: Gelechioidea \\ Diagnosis}

Vertex and frons decorated with smooth scales; labial palpus three segmented, upturned, third segment long, acute; forewing with veins R4+R5 stalked; hind tibia with dorsal surface furnished with long slender scales.
Family: Gelechiidae

Gelechiidae Stainton, 1854, Insecta Br. Lepid. Tineina, 10 (key) and 75 (spelled as Gelechidae).

Type-genus: Gelechia Hübner, (1825) 1816, Vertz. bekannter Schmett., 415.

\section{Diagnosis}

Vertex and frons covered with smooth scales; antenna smaller than $3 / 4^{\text {th }}$ length of forewing; labial palpus upturned, second segment long, acute; hindwing with veins $R_{1}$ and $S c$ united from base of wing or $R_{1}$ running into $\mathrm{Sc}$ beyond base of wing, discocellular perpendicular to long axis of wing or directed at 450 angle toward base of wing from M2, termen excavated.

\section{Subfamily: Gelechiinae}

Gelechiinae Stainton, 1854, Insecta Br. Lepid. Tineina, 10 (key) and 75 (as Gelechidae).

Type-genus: Gelechia Hübner [1825] 1816, Verz. bekannter Schmett., 415.

\section{Diagnosis}

Abdominal sternum-2 with a pair of venulae + a pair of apodemes (if a pair of venulae only, then forewing with veins $\mathrm{CuA}_{1}$ and $\mathrm{CuA}_{2}$ separated); female genitalia lacking secondary bursa from corpus bursae.

\section{Genus: Anarsia Zeller}

Anarsia Zeller, 1839, Isis, Leipzing, 190.

Ananarsia Amsel, 1959, Stuttg. Beitr. Naturk., 28: 32.

Type-species: Anarsia lineatella Zeller, 1839, Isis, Leipzing, 190.

Type-species: Tinea spartiella Schrank, 1802, Fauna Boica, 2(2):

104, by subsequent designation: Meyrick, 1925, In Wytsman, Genera Insect., 184: 153.

\section{Diagnosis}

Labial palpus second segment covered with long or small scales, the latter arranged subtriangularly, third segment relatively reduced in male, long and acute in female; antenna filiform, less than $3 / 4^{\text {th }}$ length of forewing; forewing with pterostigma between vein Sc and wing margin (costa) ending at $R_{1}, R_{4}+R_{5}$ stalked, sometimes $M_{1}+R_{4}+R_{5}$ stalked, $R_{5}$ to costa; hindwing with Rs+M stalked, $M_{3}$ and CuA connate; metathoracic legs with hair like scales on hind tibia; male genitalia with well developed socii present; gnathos absent; tegumen long or small; saccus absent or present; valvae asymmetrical, cucullus with modified scales; female genitalia with papillae anales sparsely setose; anterior apophyses rod-like, longer than posterior apophyses, broader at base; ductus bursae often coiled near corpus bursae; corpus bursae ovate or subovate in shape; signum present, crescent shaped.

\section{Anarsia tegumentus Rose and Pathania}

Anarsia tegumentus Rose and Pathania, 2003, Entomon 28(4): 330332.

Material examined:

Holotype: Male, 2.vi.1998, Forest Research Institute Campus, Dehradun, Dist. Dehradun, Uttaranchal, $700 \mathrm{~m}$, coll. P.C. Pathania Paratypes: 2 males, 2.vi.1998, Forest Research Institute Campus, Dehradun, Dist. Dehradun, Uttaranchal, 700m, coll. P.C. Pathania Larval host plant: Unknown

\section{Anarsia patulella (Walker)}

Gelechia patulella Wallker,1864, List Specimens lepid. Insects Colln Br. Mus., 29: 635.

Gelechia patulella Walsingham,1887, in Moore, Lepid. Ceylon, 3: 510.

Anarsia patulella Meyrick, 1913, J. Bombay nat. Hist. Soc., 22: 168. Anarsia patulella Meyrick,1925, in Wytsman, Genera Insect., 184: 153, nr. 17.

Anarsia patulella Caradja \& Meyrick,1935, Microlep. Kiangsu, 69. Material examined: 1 male, 3.iv.1999; 1 male, 21.iv.1999; 15 males, 8 females, 23.iv.1999; 3 males, 2 females, 25.iv.1999; 1 male, 23.vi.1999; 1 female, 17.iv.2000; 2 males, 18. iv.2002, Forest Research Institute (c) Zoo Outreach Organisation; www.zoosprint.org Manuscript 1352; Received 12 April 2005; Revised received 27 July 2005; Finally accepted 11 October 2005; Date of publication 21 December 2005 January 2006 | ISSN 0973-2535 (Print edition); 0973-2551 (Online edition) 
Campus, Dehradun, Dist. Dehradun, Uttaranchal, 700m, coll. P.C. Pathania

Distribution: India, Thailand, Sri Lanka, Taiwan and Australia (Park and Ponomarenko, 1996).

Larval host plant: Unknown

\section{Anarsia valvata Rose and Pathania}

Anarsia valvata Rose and Pathania, 2003, Entomon 28(4): 335-337. Material examined:

Holotype: Male, 10.ix.1998, University of Horticulture and Forestry, Nauni, Dist. Solan, Himachal Pradesh, $1360 \mathrm{~m}$, coll. P.C. Pathania

Paratypes: 1 male, 10.ix.1998; 3 females, 12.ix.1999, University of Horticulture and Forestry, Nauni, Dist. Solan, Himachal Pradesh, 1360m; 1 female, 17.vii.1999, Tanyhar, Dist. Mandi, Himachal Pradesh, 1120m, coll. P.C.Pathania

Larval host plant: Unknown

\section{Anarsia renukaensis Rose and Pathania}

Anarsia renukaensis Rose and Pathania, 2003, Entomon 28(4): 337339.

Material examined:

Holotype: Male, 13.iv.1999, Renuka Lake, Dist. Sirmour, Himachal Pradesh, $740 \mathrm{~m}$, coll. P.C. Pathania

Paratypes: 1 male, 13.iv.1999; 2 females, 14.iv.1999, Renuka Lake, Dist. Sirmour, Himachal Pradesh, 740m, coll. P.C.Pathania

Larval host plant: Unknown

\section{Anarsia didymopa Meyrick}

Anarsia didymopa Meyrick 1916, Exot. Microlepid., 1: 583.

Anarsia didymopa Meyrick 1925, in Wytsman, Genera Insect., 184: 154 , nr 28.

Material examined: 1 male, 1 female, 9.viii.1999; 1 male, 19.viii.1999; 1 male, 28.ix.1999; 1 male, 6.x.1999, Forest Research Institute Campus, Dehradun,Dist. Dehradun, Uttaranchal, 700m; 2 females, 12.viii.1999, Punjabi university, Patiala, Dist. Patiala, Punjab, $250 \mathrm{~m}$, coll. P.C.Pathania Old distribution: India (Pusa, Bengal) and Thailand (Park \& Ponomarenko, 1996).

Larval host plant: Capparis horrida (Fletcher, 1921).

\section{Anarsia parkae Rose and Pathania}

Anarsia parkae Rose and Pathania, 2003, Entomon 28(4): 341-343. Material examined:

Holotype: Male, 16.vii.1999, Tanyhar, Dist. Mandi, Himachal Pradesh, $1120 \mathrm{~m}$, P.C.Pathania coll.

Paratypes: 1 male, 1 female, 16.vii.1999; one male, 17.vii.1999, Tanyhar, Dist. Mandi, Himachal Pradesh, 1120m, coll. P.C. Pathania

Larval host plant: Unknown

\section{Anarsia tanyharensis Rose and Pathania}

Anarsia tanyharensis Rose and Pathania, 2003, Entomon 28(4): 343346.

Material examined:

Holotype: Male, 16.vii.1999, Tanyhar, Dist. Mandi, Himachal Pradesh, $1120 \mathrm{~m}$, coll. P.C. Pathania

Paratypes: 2 males, 16.vii.1999, Tanyhar, Dist. Mandi, Himachal Pradesh, $1120 \mathrm{~m}$, coll. P.C.Pathania

Larval host plant: Unknown

\section{Anarsia triglypta Meyrick}

Anarsia triglypta Meyrick,1933, Exot. Microlepid., 4: 354.

Material examined: 1 female, 24.iii.1998; 3 males, 1 female, 30.viii.1998; 1 male, 1 female,31.viii.1998; 3 male, 1 female, 31.ix.1998; 2 males, 13.ix.1999; 3 males, 1 female, 14.ix.1999; 1 female, 15.ix.1999; 1 female, 16.ix., 1999; 1 female, 22.ix.1999; 1 male, 1 female, 23.ix.1999; 1 female, 27.ix.1999; 2 males, 30.ix.1999; 2 females, 3.x.1999; 4 males, 6.x.1999; 3 males, 13.x.1999, Punjabi university, Patiala, Dist. Patiala, Punjab, 250m, coll. P.C. Pathania

Old distribution: India (Bihar, Pusa) (Clarke, 1969).

Larval host plant: Acacia catechu (Clarke, 1969).

\section{Anarsia veruta Meyrick}

Anarsia veruta Meyrick, 1918, Exot. Microlepid., 2: 148.

Anarsia veruta Meyrick, 1925, in Wytsman, Genera Insect., 184: 29. Material examined: 1 female, 24.vi.1999; 1 male, 26.vi.1999; 2 males, 17.iv.2000, Krishi Vishwavidalaya, Palmpur, Dist. Kangra, Himachal Pradesh, $700 \mathrm{~m}$, coll. P.C.Pathania

Old distribution: India (Bengal, Pusa) (Clarke 1969).

Larval host plant: Inga dulcis (Fletcher, 1921).

\section{Anarsia reciproca Meyrick}

Anarsia reciproca Meyrick,1920, Exot. Microlepid., 2: 300.

Material examined: 4 males, 5 females, 2.vi. 1998; 5 males, 3 females, 3.vi.1998, Forest Research Institute Campus, Dehradun, Dist Dehradun, Uttaranchal, 700m; 5 females, 27.vi.1998, Tanyhar, Dist. Mandi, Himachal Pradesh, $1120 \mathrm{~m}$, coll. P.C.Pathania

Old distribution: India (Madras), Coimbatore (Clarke, 1969).

Larval host plant: Unknown

\section{Genus: Stegasta Meyrick}

Stegasta Meyrick, 1904, Proc. Linn. Soc. N.S.W., 29: 258 (key), 313. Type-species: Stegasta variana Meyrick, 1904, Proc. Linn. Soc. N.S.W., 29: 313 (key), 314, by original designation.

\section{Diagnosis}

Labial palpus upturned, third segment long and acute; antenna filiform, about $3 / 4^{\text {th }}$ length of forewing, creamish and fuscous band alternatively; forewing with expansible pencil of creamy white scales in a costal fold on the ventral surface in males and by the characteristic pattern of the forewing, vein $R_{4}+R_{5}$ stalked, $M_{3}+C u A_{1}$ stalked; hindwing with veins Rs+M stalked, Rs to costa, $M_{1}$ to termen, $M_{3}$ free; male genitalia with uncus bifid; socii well developed; gnathos absent; saccus absent; valvae rather slender, broader toward base, expanded distally, numerous strong setae on inner surface of distal part, with a strong spine-like projection near base of cucullus; aedeagus tubular with one or two long horn shaped lobes; cornutus present or absent; female genitalia with papillae anales large, broad, densely setose anterior apophyses rod-like, broader at base; corpus bursae membraneous, bulb-shaped; signum sickle-shaped

\section{Stegasta comissata Meyrick}

Stegasta comissata Meyrick, 1923, Exot. Microlepid., 3: 18.

Material examined: 1 female, 15.ix.1998; 1 female, 29.ix.1998; 3 females, 28.x.1998; 1 male, 7.xi.1998; 1 male, 10.iii.1999; 1 male, 12.x.1999; 2 males, 4.xi.1999; 4 males, 6.iii.2000; 1 male, 9.iii.2000; 2 males, 10.iii.2000; 1 female, 16.iii.2000; 5 males, 28.iii.2000; 2 males, 2 females, 5.iv.2000; 2 males, 10.iv.2000; 2 males, 16.v.2000; 2 males, 17.v.2000; 18 males, 23.ix.1999, Forest Research Institute Campus, Dehradun, Dist. Dehradun, Uttaranchal, 700m; 3 males, 18.v.2000; 2 females, 24.v.2000,Punjabi University, Patiala, Dist. Patiala, Punjab, 250m; 3 males, 2 females, 9.xi.2001, Jammu University Campus, Dist. Jammu, 350m, coll. P.C. Pathania

Old distribution: Brazil, Obidos, Santarem, Parintins, Manaos (Clarke, 1969a).

Larval host plant: Unknown.

\section{Stegasta $\mathrm{sp}$}

Material examined: 2 males, 13.iv.1998; 1 male, 1 female, 10.ix.1998; 6 males, 2 females, 5.iv.2000; University of Horticulture and Forestry, Nauni, Dist. Solan, Himachal Pradesh, 1360m; 14 males, 4 females, 13.iv.1999, 9 males, 2 females, 21.iv.2000, Renuka Lake, Dist. Sirmour, Himachal Pradesh, 740m; 2 males, 16.ix.2001, Dharampur, Dist. Solan, Himachal Pradesh, 1500m; 6 males, 27.v.2001, Krishi Vishwavidalaya, Palmpur, Dist. Kangra, Himachal Pradesh, 700 m, coll. P.C.Pathania Larval host plant: Unknown.

\section{Genus: Hypatima Hübner}

Hypatima Hübner, [1825], Verz. bekannter Schmett., 415.

Allocota Meyrick, 1904, Proc. Linn. Soc. N.S.W., 29: 258. Typespecies: Allocota simulacrella Meyrick, 1904, Proc. Linn. Soc. 
N.S.W., 29: 420.

Allocotaniana Stand, 1913, Arch. Nat., 79(42): 43. Type-species: Allocota simulacrella Meyrick, 1904, Proc. Linn. Soc. N.S.W., 29: 420.

Chelaria Haworth, 1828, Lepid. Br., 526. Type-species: Chelaria conscripta Haworth, 1828, Lepid. Br., 526.

Cymatomorpha Meyrick, 1904, Proc. Linn. Soc.N.S.W., 29: 258. Typespecies: Cymatromorpha euplecta Meyrick, 1904, Proc. Linn. Soc. N.S.W., $29: 57$ (key) 411.

Episacta Turner, 1919, Proc. R. Soc. Qd., 31: 161. Type-species: Chelaria discissa Meyrick, 1916, Exot. Microlepid., 1: 581.

Semodictis Meyrick, 1909, Ann. Trans. Mus., 2: 16. Type-species: Semodictis tetraptial Meyrick, 1909, Ann. Transv. Mus., 2: 16.

Type-species: Tinea conscriptella Hübner, 1805, Samml, eur. Schmett., 8: pl.41. fig.283 by sebsequent designation by Walsingham \& Durrat, 1909, Entomologists mon. Mag., 45: 48.

\section{Diagnosis}

Labial palpus long, upturned, second segment with a long loose hair like scaletuft, the latter divided into two parts, third segment long, acute, usually with a rough scaletuft dorsally; antenna long, $3 / 4^{\text {th }}$ length of forewing; metathoracic leg with hair like scales on hind tibia; forewing elongate, veins $R_{4}+R_{5}$ out of the stalk of $M_{1}$ or $M_{1}$ free, $R_{5}$ to costa; hindwing with Rs+M stalked, Rs to costa, $M_{1}$ to termen, $M_{3}$ and $C u A_{1}$ connate or stalked, a brush of long hair pensil between veins $\mathrm{CuA}_{1}$ and $\mathrm{CuA}_{2}$ directed toward base of wing present or absent; male genitalia uncus broader at base narrowed towards apex, bearing small hair like setae, socii lacking; gnathos stout; tegumen with setose lobe at base; vinculum with a pair of processes, symmetrical or asymmetrical; aedeagus broader basally, apically pointed, bend at middle; female genitalia with papillae anales sparsely setose; ostium bursae with a heavily sclerotized ring shaped structure posteriorly; ductus bursae long and coiled; corpus bursae ovate or subovate shaped; signum small or large, kite-shaped.

\section{Hypatima tephroptila (Meyrick)}

Chelaria tephroptila Meyrick, 1931, Exot. Microlepid., 4: 70

Material examined: 2 females, 23.iv.1999; 2 males, 2 females, 25.iv.1999, Forest Research Institute Campus, Dehradun, Dist. Dehradun, Uttaranchal, $700 \mathrm{~m}$; coll. P.C.Pathania

Old distribution: India (Bombay, Mahableshwar) (Clarke, 1969). Larval host plant: Unknown

\section{Hypatima vinculata Pathania and Rose}

Hypatima vinculata Pathania and Rose, 2003, Pest Management and Economic Zoology, 11(2): 166-168.

Material examined:

Holotype: Male, 29.viii.1998, Punjabi university, Patiala, Dist. Patiala, Punjab, 250m, coll. P.C.Pathania

Paratypes: 2 males, 27.ix.1999, Punjabi university, Patiala, Dist. Patiala, Punjab, 250m, coll. P.C.Pathania

Larval host plant: Unknown

\section{Hypatima spathota (Meyrick)}

Chelaria spathota Meyrick, 1913, J. Bombay nat. Hist. Soc., 22: 165. Chelaria spathota Gaede, 1937, Lepid. Cat., : 414.

Chelaria spathota Clarke, 1969, Cat. Type Specimens Microlepid. $B M N H .$, (6): 426, fig. 3.

Material examined: 2 females, 25.v.1998; 2 females, 13.iv.1999, Renuka Lake, Dist. Sirmour, Himachal Pradesh, 740m; 5 males, 23.iv.1999; 2 males, 25.iv.1999; 1 female, 3.iv.1999, Forest Research Institute Campus, Dehradun, Dist. Dehradun, Uttaranchal, 700m; coll. P.C.Pathania

Old distribution: India (Khasi Hills, Konkan) (Park, 1995).

Larval host plant: Unknown

\section{Subfamily: Dichomeridinae}

Dichomeridinae Hampson, 1918, Novit. zool., 25: 386.

Type-genus: Dichomeris Hübner, 1818, Zutr. Samml. exot. Schmett.,
1: 25 .

\section{Diagnosis}

Abdominal sternum-2 with a pair of venulae only; forewing usually with $\mathrm{CuA}_{1}$ and $\mathrm{CuA}_{2}$ stalked and directed posteriorly from end of cell; female with secondary bursa arising from corpus bursae.

\section{Genus: Hypelictis Meyrick}

Hypelictis Meyrick, 1905, J. Bombay nat. Hist. Soc., 16: 600.

Type-species: Hypelictis acrochlora Meyrick, 1905, ibidem., 16: 600.

\section{Diagnosis}

Labial palpus long, arm-like, strongly upturned, covered with scales; antenna long, filiform, $3 / 4^{\text {th }}$ length of forewing; metathoracic leg with hair like scales on hindtibia; forewing with veins $R_{4}+R_{5}$ very long stalked, $R_{5}$ to costa, CuA $+C u A_{2}$ stalked; hindwing with vein $R_{1}$ running into $\mathrm{Sc}, \mathrm{Rs}+\mathrm{M}_{1}$ connate, $\mathrm{R}_{5}$ to apex, $\mathrm{M}_{1}$ to termen, $\mathrm{M}_{3}+\mathrm{CuA}$, stalked, $1 A+2 A$ forked at base; male genitalia with uncus narrowed towards apex, setose; socii absent; gnathos small, hook-like; two setose, one $\mathrm{Y}$-shaped and one elongated arm like lobe on vinculum; valvae small, broader distally, inner surface densely setose; aedeagus long, with long plate and cornutus present; female genitalia with papillae anales sparsely setose; anterior apophyses absent; ostium bursae broad; corpus bursae large, sclerotized, with hair-like projection near basal half; ductus seminalies arising near middle of corpus bursae; signum absent or present.

\section{Hypelictis acrochlora Meyrick}

Hypelictis acrochlora Meyrick, 1905, J. Bombay nat. Hist. Soc., 16: 600; Meyrick, 1925, In Wytsman, Genera Insect., 184, p.110, nr.2.

Material examined: 2 males, 22.iv.1999; 2 males, 23.iv.1999, 2 females, 18.iv.2000, Forest Research Institute Campus, Dehradun, Dist. Dehradun, Uttaranchal, $700 \mathrm{~m}$; coll. P.C. Pathania

Old distribution: Maskeliya, Ceylon (Clarke, 1969).

Larval host plant: Unknown

\section{Genus: Dichomeris Hübner}

Dichomeris Hübner, 1818, Autr. Samml. exot. Schmett., $1: 25$.

Type-species: Dichomeris ligulella Hübner, 1818, ibidem., 1: 25, pl. [25], figs 143,144 , by subsequent designation: Walsingham, 1911, Biologia cent.-am., Zool., Lepid. Heterocera, 4: 87.

\section{Diagnosis}

Labial palpus long, upturned, with or without brush of scales, the latter directed anteriorly, third segment long and acute; antenna filiform, less than $3 / 4^{\text {th }}$ length of forewing; hindtibia of the metathoracic leg with small hair-like scales; forewing with vein $R_{4}+R_{5}$ stalked, $R_{5}$ to costa or apex, $\mathrm{R}_{5}$ sometime absent, $\mathrm{CuA}_{1}+\mathrm{CuA}_{2}$ stalked; hindwing with vein $R s+M_{1}$ stalked, $M_{3}+C u A_{1}$ stalked or connate; male genitalia with uncus dialated distally; culcitula present; gnathos hook-like; tegumen with setose lobe at base; sicae symmetrical or asymmetrical present; valvae long, cucullus bearing setae on the inner surface; aedeagus with rod-like lateral lobe present; female genitalia with anterior apophyses very short, stout; corpus bursae densely spiculose in part; heavily sclerotized ring near base of ductus seminalis; lacking sclerotized signum.

\section{Dichomeris sicaellus Pathania and Rose}

Dichomeris sicaellus Pathania and Rose, 2003, Him. J. Env. Zool., 17(1): 10-11.

Material examined:

Holotype: Male, 5.iv.2000, University of Horticulture and Forestry, Nauni, Dist. Solan, Himachal Pradesh, 1360m, coll. P.C. Pathania Larval host plant: Unknown

\section{Dichomeris sicaellus acuminata (Staudinger)}

Mesophleps (?) acuminatus Staudinger, 1876, Ent. Ztg. Stett., 37: 148.

Hyposolophus ianthes Meyrick, 1887, Trans. ent. Soc. Lond., 273. 
Dichomeris ianths; Gaede, 1937, Lepid. Cat., 79: 434; Issiki, 1957, In. Heterocerorum Japonicorum Coloribus Naturalibus.: 42, pl. 6, fig. 176; Clarke, 1969, Cat. Type Specimens

Microlepid. BMNH,: 27, pl. 13, fig. 3; Moriuti, 1982, Moths of Japan,: 1/284, 2/215, pl. 13, fig. 33.

Ypsolophus rusticus Walsingham, 1891, Proc. zool. Soc. Lond.,: 525.

Ypsolopus lotellus Constant, 1893, Ann. Soc. ent. France, 62: 398. Ypsolophus ammoranthus Meyrick, 1904, Proc. Linn. Soc. N.S.W., 29: 430.

Ypsolophus ochrophanes Meyrick, 1907, J. Bombay nat. Hist. Soc., 17: 981.

Dichomeris acuminatus; Zimmermann, 1978, Insects of Hawaii, 9(2): 1706, figs. 1262-1267.

Dichomeris acuminata; Hodges, 1986, The Moths of America north Mexico, 7: 38, pl. 4, figs. 1, text fig. 9; Park \& Hodges 1995, Inst. Koreana, 12: 28

Material examined: 1 male, 11.ix.1998,University of Horticulture and Forestry, Nauni, Dist. Solan, Himachal Pradesh, 1360m; 3 males, 24.ix.1999, Forest Research Institute Campus, Dehradun, Dist. Dehradun, Uttaranchal, $700 \mathrm{~m}$; coll. P.C. Pathania

Old distribution: Taiwan, Japan, Widely distributed in oriental and Australian region (Park and Hodges, 1995).

Larval host plant: Medicago sativa, Camopis sp., Desmodium gyroides, Cajanus cajan, Sebania Serica and Tephrosia sp. (Park \& Hodges, 1995).

\section{Dichomeris sicaellus rasilella (Herrich-Schaffer)}

Anacampsis rasilella Herrich-Schaffer, 1855, Schmett. Eur., 5: 202. Brachmia rasilella Rebel, 1901, Famil. Pyralidae-Micropterygidae.: 157.

Gompphocrates rasilella Meyrick, 1925, in Wytsman, Genera Insect: 184.

Uliaria rasilella Dumont, 1921, Bull. Soc. ent. France., 329; Issiki, 1957, In. Heterocerorum Japonicorum Coloribus Naturalibus.: 39 , pl. 5, fig. 161; Moriuti, 1982, Moths of Japan, 1/286, 2/215, pl. 13: 47; Park, 1983, Insecta 9: 505, fig. 167, Park \& Hodges 1995, Inst. Koreana, $12: 52$.

Material examined: 1 male, 12.ix.1999; 3 males, 5.iv.2000, University of Horticulture and Forestry, Nauni, Dist. Solan, Himachal Pradesh, 1360m; 2 females, 13.iv.1999, Renuka Lake, Dist. Sirmour, Himachal Pradesh, $740 \mathrm{~m}$; coll. P.C. Pathania

Old distribution: Taiwan, Japan, Korea, Chiana, Russia, Europe (Park \& Hodges, 1995)

Larval host plant: Unknown

\section{Genus: Helcystogramma Zeller}

Helcystogramma Zeller, 1877, Horae Soc. ent. ross., 13: 369.

Ceratophora Heinemann, 1870, Schmett. Dtl. Schweiz, (2)(1): 325. Type-species: Recurvaria rufescens Haworth, 1828, Lepid. Br., 555. Teuchophanes Meyrick, 1914, Trans. ent. Soc. Lond.: 274. Typespecies: T. leucopleura Meyrick, 1914, Trans. ent. Soc. Lond., 274. Psamathoscopa Meyrick, 1937, Exot. Microlepid. 5: 96. Type-species: Onebala simplex Walsingham, 1900, Bull. Lpool. Mus., 3: 2.

Anathyrsotis Meyrick, 1939, Trans. R. ent. Soc. Lond., 89 : 55. Typespecies: A. ceriochranta Meyrick, 1939, Trans. R. ent. Soc. Lond., $89: 55$.

Type-species: Gelechia (Helcystogramma) obseratella Zeller, 1877, Horae Soc. ent. ross., 13: 371 , pl. 5, fig. 127, by susequent designation: Meyrick, 1910, Entomologist's mon. Mag., 46: 282.

\section{Diagnosis}

Labial palpus long, sickle shaped, third segment long and acute; antenna filiform approaching at $3 / 4^{\text {th }}$ length of forewing; forewing with veins $R_{4}+R_{5}$ stalked, $R_{4}$ to costa, $R_{5}$ to termen or apex, CuA $+C u A_{2}$ stalked, discal cell closed; hindwing somewhat quadrate, vein Rs+M1 stalked, Rs to apex, $\mathrm{M}_{1}$ to termen, $\mathrm{M}_{3}+\mathrm{CuA}$ stalked, CuP vestigial or absent, discal cell open; male genitalia with uncus broader at apex, narrowed basally, small hair on inner surface; socii absent; gnathos strongly sclerotized, hook-like, tegumen hood-like, valvae with paired setose lobe arising from lateral arms, valvae long, broader distally, setose densely; saccus present; coremata present or absent; aedeagus broader basally, apically acute; female genitalia with papillae anales sparsely setose; anterior and posterior apophyses almost equal in length; ductus seminalis arising from right posterior of corpus bursae; corpus bursae with slightly sclerotized portion at juntion of ductus seminalis.

\section{Helcystogramma hibisci (Stainton)}

Gelechia (?) hibisci Stainton, 1859. Trans. ent. Soc. Lond., (2)5, p. 117.

Onebala Hibisci: Meyrick, 1925, in Wytsman, Genera Insect., p. 138; Gaede, 1937 Lepid. Cat., p. 377.

Gelechia (Helcystogramma) obseratella zeller, 1877, Horae Soc. ent. Ross., 13, p. 371.

Croesophora eudela Turner, 1919, Proc. Roy. Soc. Queensland, 31, p. 160.

Material examined: 2 females, 13.iv.1999, Renuka Lake, Dist. Sirmour, Himachal Pradesh, 740m; 1 male, 23.iv.1999, Forest Research Institute Campus, Dehradun, Dist. Dehradun, Uttaranchal, 700m; 2 males, 19.viii.1999, Punjabi university, Patiala, Dist. Patiala, Punjab, 250m, three males, 5.iv.2000, University of Horticulture and Forestry, Nauni, Dist. Solan, Himachal Pradesh, 1360 m, coll. P.C. Pathania

Distribution: India (Kolkata), S. China, Taiwan, Sri Lanka, Java and Australia (Park \& Hodges, 1995).

Larval host plant: Hibiscus exculentus (Fletcher, 1921), Okra (Butani and Jotwani, 1984).

\section{Helcystogramma clarkei Rose and Pathania}

Helcystogramma clarkei Rose and Pathania, 2003, Pb.Univ. Res. J.(Sci), 53: 84-86.

Material examined:

Holotype: Male, 23.x.1998, Punjabi University, Patiala, Dist. Patiala, Punjab, 250m, coll. P.C. Pathania

Paratypes: 2 males, 1 female, 23.iv.1999, Forest Research Institute Campus, Dehradun, Dist. Dehradun, Uttaranchal, 700m; 1 female, 21.vii.1998, 2 males, 3.x.1999; 1 male, 4.x.1999; 1 male, 5.x.1999; 1 male, 13.x.1999; 1 male, 15.xii.99, Punjabi University, Patiala, Dist. Patiala, Punjab, $250 \mathrm{~m}$, coll. P.C. Pathania

Larval host plant: Unknown

\section{Helcystogramma uedai Rose and Pathania}

Helcystogramma uedai Rose and Pathania, 2003, Pb.Univ. Res. J.(Sci), 53: 86-88.

Material examined:

Holotype: Male, 11.iv.1999,Renuka Lake, Dist. Sirmour, Himachal Pradesh, $740 \mathrm{~m}$, coll. P.C. Pathania

Paratypes: 3 females, 6.ix.1998, Punjabi university, Patiala, Dist. Patiala, Punjab, 250m; 1 female, 3.iv.1999, Forest Research Institute Campus, Dehradun, Dist. Dehradun, Uttaranchal, 700m; 1 male, 5.vi.1999, Tanyhar, Dist. Mandi, Himachal Pradesh, 1120m; 1 male, 11.iv.1999; two females, 21.iv.2000, Renuka Lake, Dist. Sirmour, Himachal Pradesh, $740 \mathrm{~m}$; coll. P.C. Pathania

Larval host plant: Unknown

\section{Discussion}

The main characteristic of the family Gelechiidae are "Vertex and frons covered with smooth scales, antennae smaller than $3 / 4^{\text {th }}$ length of forewings, labial palpus upturned, second segment long, acute, hindwing with veins $\mathrm{R}_{1}$ and $\mathrm{Sc}$ united from base of wing or $R_{1}$ running into Sc beyond base of wing, discocellular perpendicular to long axis of wing or directed at 45 degree angle toward base of wing from $\mathrm{M}_{2}$, termen excavated.

In the subfamily Gelechiinae, Anarsia Zeller with labial palpus second segment beset with small sacles, the latter arranged 
subtriangularly, third segment reduced in male, male genitalia with valvae asymmetrical, cucullus with modified scales on the inner surface, tegumen longer and socii present. Stegasta Meyrick is being characterized by the presence of creamishwhite scales in a costal fold on the ventral surface of the forewing, male genitalia with uncus bifid, aedeagus tubular in shape and female genitalia with corpus bursae bulb-like. In Hypatima Hübner the labial palpus with second segment beset with relatively longer scales, directely anteriorly, third segment well developed in male and male genitalia with socii absent.

In the subfamily Dichomeridinae the genus Hyplectis Meyrick is characterized with labial palpus arm-like, second and third segment scales and Dichomeris Hübner with forewing vein $\mathrm{R}_{5}$ to costa or apex (absent in rasilella Herrich-Schaffer) and male genitalia with culcitula, sicae present and aedeagus with well developed lateral lobes but in genus Helcystogramma Zeller forewing vein $\mathrm{R}_{5}$ to termen and male genitalia with out culcitula and sicae and aedeagus without such lobes.

\section{REFERENCES}

Butani, D.K. and M.G. Jotwani (1984). Insects in vegetables. Periodical Expert Book Agency, New Delhi, 356pp.

Chitkara, M.G. (1998). Encyclopaedia of Ecology. Environment and Pollution 6: 1-339.

Clarke, J.F.G. (1969a). Catalogue of the type specimens of microlepidoptera in the British Mus. (Natural History) described by Edward Meyrick, Trustees of the British Museum (Natural History), London, VI: $537 \mathrm{pp}$.

Clarke, J.F.G. (1969b). Catalogue of the type specimens of microlepidoptera in the British Mus. (Natural History) described by Edward Meyrick, Trustees of the British Museum (Natural History), London, VII: $531 \mathrm{pp}$.

Common (1970). Lepidoptera, The Insects of Australia. chapter $3 \mathrm{~b}$. CSIRO, Divn. Entomology, Melbourne.

Fletcher, T.B. (1921). Life history of Indian Insects, Microlepidoptera. Mem. Dep. Agric., India 6: 217.

Gaede, M., (1937). Catalogue of Gelechiidae. Lepidopterorum catalogus 79: 1630

Hodges, R.W. (1998). Gelechioidea, pp.131-158. In: Handbook of Zoology.

Klots, A.B. (1970). Taxonomists Glossary of Genitalia in Insects. Munksgasard, Copenhagen Lepidoptera, 115-139pp in Tuxen.

Landry, J.F. and B. Landry (1994). A technique for setting and mounting microlepidoptera. Journal of Lepidoptera Society 48(3): 205227.

Lindquist, O.H. (1956). A technique for pinning and spreading small microlepidoptera. Canadian Entomology 138(1): 24-25.

Meyrick, E. (1905). Descriptions of Indian Microlepidoptera. Journal of the Bombay Natural History Society 16: 580-619.

Meyrick, E. (1907). Descriptions of Indian Microlepidoptera. Journal of the Bombay Natural History Society 17: 730-754, 976-994.

Meyrick, E. (1908a). Descriptions of Indian Microlepidoptera. Journal of the Bombay Natural History Society 18: 137-160, 437-460, 613638, 806-832,

Meyrick, E. (1908b). New Micro-Lepidoptera from India and Burma. Records of Indian Museum 12: 3295-400.

Meyrick, E. (1909). Descriptions of Indian Microlepidoptera. Journal of the Bombay Natural History Society 19: 410-437, 582-607.

Meyrick, E. (1910). Descriptions of Indian Microlepidoptera. Journal of the Bombay Natural History Society 20: 143-168, 435-462, 706736.

Meyrick, E. (1911). Descriptions of Indian Microlepidoptera. Journal of the Bombay Natural History Society 21: 104-131, 852-877.
Meyrick, E. (1912-1916). Exot. Microlepid., 1: 1-640.

Meyrick, E. (1913). Description of Indian Microlepidoptera. Journal of the Bombay Natural History Society 22: 771-781.

Meyrick, E. (1914). Descriptions of Indian Microlepidoptera. Journal of the Bombay Natural History Society 23: 118-130.

Meyrick, E. (1916-1923). Exot. Microlepid., 2: 1-640.

Meyrick, E. (1923-1930). Exot. Microlepid., 3: 1-640.

Meyrick, E. (1930-1936). Exot. Microlepid., 4: 1-642.

Mikkola, K. (1986). Tower spreading, a handy method for provisional field preparation for microlepidoptera. Not. Entomol., 66: 101-102.

Park, K.T. (1995). Gelechiidae of Taiwan. 1. Review of Anarsia, with descriptions of four new species (Lepidoptera: Gelechioidea). Tropical Lepidoptera 6(1): 55-66.

Park, K.T. and R.W. Hodges (1995). Gelechiidae (Lepidoptera) of Taiwan III. Systematic Revision of the genus Dichomeris in Taiwan and Japan. Institute of Koreana 12: 1-101.

Park, K.T. and M. Omelko (1994). Two new species of Genus Stegasta (Lepidoptera, Gelechiidae). Japan Journal of Entomoly 62(4): 867871.

Park, K.T. and M.G. Ponomarenko (1996). Genus Anarsia Hubner (Lepidoptera: Gelechiidae) from Thailand, with description of nine new species. Institute of Koreana 13: 39-58.

Robinson, G.S. (1976). The preparation of slides of Lepidoptera genitalia with special reference to microlepidoptera. Entomology Gazeteer 27(2): 127-132.

Robinson, G.S., K.R. Tuck and M. Shaffer (1994). A Field Guide to the Smaller Moths of South-East Asia. Malaysian Nature Society, Malasia $308 \mathrm{pp}+32 \mathrm{pls}$.

Tagestad, A.D. (1974). A technique for mounting microlepidoptera. Journal of Kansas Entomolgy Society 47: 26-30.

Ueda, T. (1995). The genus Helcystogramma (Lepidoptera: Gelechiidae) of Japan. Japan Journal of Entomology 63(2): 377-387.

Zimmerman, E.C. (1978). Microlepidoptera in Hawaii. Vol. 9. University Press of Hawaii, Honololu, xviii+1903pp.

\section{ACKNowledgement}

We thank to the Ministry of Environment and Forests (GOI), New Delhi, for funding the project on moths and to the Vice Chancellor, Punjabi University, Patiala for providing facilities to run the coordinating centre on Microlepidoptera under an All India Coordinated Project on Taxonomy (AICOPTAX). We also thank Dr. K.T. Park (Korea) for sending much needed literature and valuable suggestions during the course of these studies. 